\title{
NOUVELLES FOURMIS DU CONGO BELGE
}

\author{
DU \\ MUSÉE DU CONGO BELGE, A TERVUEREN \\ PAR
}

le Dr F. SANTSCHI

\section{I. - Paltothyreus tarsatus F.}

Cette espèce varie beaucoup, mais certains de ces caractères ont une fixité qui correspond aux principales régions du Continent africain. On peut en distinguer les variétés suivantes :

\section{Var. tarsatus $F_{A B}$.}

Les stries des côtés de la tête sont plus fortes, continues et plus régulières; elles sont aussi plus obliques; celles qui passent près du bord externe de l'œil atteignent le bord postérieur de la tête vers son quart externe; mandibules bien striées, l'écaille lisse, le sommet largement arrondi, presque transversal; la taille grande chez les sexués.

ㅇ. Longueur, $25 \mathrm{~mm}$. (avec les mandibules); aile supérieure, $15 \mathrm{~mm}$. Largeur de la tête, $4.5 \mathrm{~mm}$.; maxima du thorax, $4.2 \mathrm{~mm}$.; du gastre, $4.4 \mathrm{~mm}$.

$\sigma^{T}$. Longueur totale, I9 $\mathrm{mm}$; de l'antenne, I3-I4 mm.; de l'aile antérieure, $\mathrm{I} 3.5^{-1} 4 \mathrm{~mm}$.

Largeur du thorax, $3.4 \mathrm{~mm}$.; du gastre, $3.7 \mathrm{~mm}$.

Sénégal : type de Fabricius ơ.

Côte d'Ivoire : Dimbroko (Le Moult), §̊; Jacqueville et Grand- 


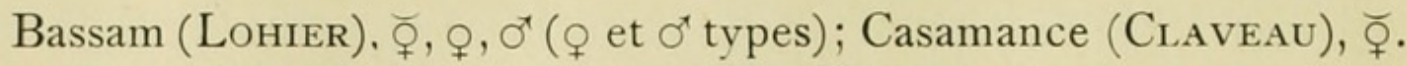
Congo français : Fort-Crampel (Le Moult), Ф̆.

\section{Var. mediana n. var.}

Diffère de la variété type par les stries des côtés de la tête qui sont plus irrégulières, interrompues par une nombreuse ponctuation plus fine et plus longitudinale. La face postérieure de l'écaille est plus ou moins striée chez la $\&$; son sommet forme une courbe plus étroite et plus fortement arquée chez $\bar{q}$ et $q$. La taille des sexués est moins avantageuse.

‥ Longueur, $23-2+\mathrm{mm}$. Largeur de la tète, $4 \cdot \mathrm{I}-4 \cdot 3 \mathrm{~mm}$.; du thorax, $3.8 \mathrm{~mm}$.

$\sigma^{x}$. Longueur totale, $15 \mathrm{~mm}$; ; antennes, ${ }^{1} 3^{-14} \mathrm{~mm}$. Largeur du thorax, $3.4 \mathrm{~mm}$.; du gastre, $3.4 \mathrm{~mm}$., chez les exemplaires du Gabon.

$\sigma^{x}$. Longueur, I4 mm.; aile, II $\mathrm{mm}$. Largeur du thorax, 3.I mm.; du gastre, 3.I mm. (Brazzaville).

Congo français : Brazzaville (Weiss),, , $\subsetneq$ types (? ơ); Ubanghi (R. P. Augustin); Borda (P. Charleux).

Benguela : Cucala (CRUCHET), $q$ (exemplaires plus grands).

Cameroun : Molundu (Reichensperger).

\section{Var. subopaca n. var.}

Le dessus du gastre est en grande partie sculpté parfois entièrement de réticulations transversales et submat. L'écaille est striée derrière et au sommet Le devant de la tête, les côtés du thorax, les appendices, plus ou moins roussâtres. Taille en général plus petite mais variable.

․ Longueur totale, $2 \mathrm{I}-24 \mathrm{~mm}$; aile supérieure, $\mathrm{I} 3 \mathrm{~mm}$. Largeur de la tête, $3 \cdot 3-4 \mathrm{~mm}$; du thorax, $3 \cdot 3-4 \mathrm{~mm}$

ф. . Longueur, $17 \mathrm{~mm}$.

Gabon : Sam-Kito (F. FAure),, , Ф๐.

\section{Var. delagoensis Ем.}

Taudis que les trois variétés précédentes ont un sillon bien dégagé au milieu de l'épistome, chez la variété delagoensis (et les deux variétés suivantes) le sillon est plus ou moins comblé par une saillie longitudinale. Mandibules lisses ou très faiblement striolées. Stries latérales de la tête comme chez mediana. 
Delagoa (Liegme); Rhodesia (G. Arnold).

Afrique orientale allemande : Kilimandjaro (Alluaud) et Pangani (REICHENSPERGER).

Var. striatidens n. var.

Épistome comme chez delagoensis, mais les dents fortement striées. Stries latérales de la tête plus fortes, comme chez le type.

Afrique orientale anglaise : Kibwey (Alluaud),,, .

\section{Var. robusta n. var.}

Partie médiane de l'épistome large et plane, fortement striée avec une saillie médiane. Mandibules striées. Écailles striées derrière. Striation des côtés de la tête plus ou moins effacée. Taille plus robuste.

ㅇ. Longueur, $29 \mathrm{~mm}$.; ailes antérieures, $17 \mathrm{~mm}$. Largeur de la tête, $5 \mathrm{~mm}$.; du thorax, $4.5 \mathrm{~mm}$.; du gastre, $5 \mathrm{~mm}$.

Somalie : Buarsangueli (Revori).

Fred. Smith (I 858 ) décrit, sous le nom de Pachycondyla simillima, une $q$ de Paltothyreus tarsatus, dont le sommet de l'écaille est légèrement échancré; c'est peut-être une variété locale (Cape Colony), mais je ne la connais pas en nature.

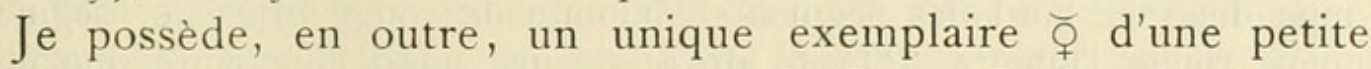
§ Paltothyreus tarsatus provenant de la Côte d'Ivoire; l'épistome est sillonné au milieu comme le type; la tête aussi fortement striée, mais les mandibules entièrement lisses. C'est peut-être aussi une variété locale.

\section{CLEF DES VARIÉTÉS.}

I. Partie médiane de l'épistome enfoncée en sillon (Afrique occidentale). 2

- Ce sillon est tout ou en partie comblé par une saillie longitudinale (Afrique orientale).

2. Côtés de la tête fortement striés; écaille peu arquée au sommet.

P. tarsatus $\mathrm{FAB}$.

- Stries latérales de la téte moins régulières; convexité plus forte du sommet de l'écaille. . 
3. Gastre et sommet de l'écaille luisant lisse, à ponctuation espacée, plus robuste . . . . . . . . . . . . . var. mediana $\mathrm{n}$.

- Gastre en grande partie sculpté, taille de la $q$ plus petite, var. subopaca $\mathrm{n}$.

4. Mandibules lisses ou très faiblement striées (Afrique du Sud-Est),

- Mandibules fortement striées (Afrique orientale)

var. delagoensis Ем.

5. Taille plus petite de la $\&$, stries latérales de la tête plus fortes,

var. striatidens n.

- Taille plus grande, côtés de la tête faiblement striés, plutôt ponctués. var. robusta $\mathrm{n}$. v.

\section{2. - Pachycondyla (Bothroponera) gabonensis ANDRÉ var. robustior $\mathrm{n}$. var.}

б.. Long. : 9-9.5 mm. Appendices plus sombres que le bord des segments du gastre. Rides de la tête très divergentes tout le long de la ligne médiane. Les fossettes du gastre sont très allongées et lui donnent un aspect strié, du reste, comme le type, mais beaucoup plus robuste.

Congo belge : Banalia, i 2-XII ( $D^{r}$ Bequaert, $\left.n^{\circ} 96\right)$.

\section{3. - Pachycondyla (Bothroponera) armata n. sp.}

ํ. Long. : 8-9.5 mm. Noire. Mandibules, épistome, lobes frontaux, appendices et bord des segments abdominaux rouge brunâtre, segment apical rouge jaunâtre. Pilosité dressée fauve aussi abondante que chez gabonensis ANDRÉ. Mandibules lisses avec quelques points. Tête striée réticulée, les stries médianes sont parallèles, les plus externes divergentes, celles qui partent de l'extrémité postérieure des arêtes frontales atteignent le bord postérieur de la tête vers son cinquième externe. Les angles postérieurs et les côtés sont plus réticulés que striés. Stries occipitales lisses avec leurs intervalles lisses. Thorax et face antérieure de l'écaille grossièrement réticulés-ridés, fossulés et luisants dans le fond des fossettes. Face déclive de l'épinotum lisse et luisante. Ponctuation pilifère du gastre courte avec l'intervalle luisant et faiblement chagriné, presque lisse sur le premier segment, plus fortement ponctué et moins luisant sur les segments suivants.

Tête presque carrée, le bord postérieur un peu concave. Les yeux sont aussi grands que l'espace qui les sépare du bord antérieur de la tête et occupent presque le quart de la longueur des côtés; sillon frontal aussi long que les arêtes frontales. L'épistome forme un lobe tronqué devant sans sillon ni dents à son bord antérieur; le milieu est luisant, les côtés réticulés 
rugueux. Mandibules étroites, une dent apicale et une basale avec deux sinuosités intermédiaires sur le bord terminal qui n'est pas très distinct du bord interne. Le scape dépasse légèrement le bord occipital; sauf le dernier, les articles du funicule sont plus larges que longs. Thorax un peu convexe. Les dents épinotales courtes, aussi larges que longues. Écaille épineuse, comme chez gabonensis. Les dents externes un peu divergentes et plus longues que chez la var. slriatidens SANTS., mais bien moins longues que $P$. Sveni For. Gastre assez étroit.

\section{Congo belge : Kitempuka (GÉRARD), I ơ.}

Cette espèce se rapproche de P. Sveni par son épistome inerme, mais bien différente de cette espèce et de gabonensis, par la sculpture de son abdomen.

\section{4. - Ponera inaudax n. sp.}

ð઼. Long. : $2 \mathrm{~mm}$. Jaune roussâtre foncé, appendices jaunes. Lisse avec une très fine ponctuation. Un peu luisante (moins luisante que chez Abeillei Em., plus que chez caca SANTS.). Quelques poils dressés, très courts, sur le gastre. Pubescence moyenne comme chez Abeillei.

Tête rectangulaire, plus longue que large (comme chez cæca), avec les côtés arqués, le bord postérieur à peine échancré; aveugle. Un sillon frontal dépasse un peu le tiers antérieur de la tête. Épistome très court, tronqué, avec une très légère carène médiane. Les mandibules luisantes ont un bord terminal plus court que celui de caca, armé de 5 denticules. Le scape assez épais n'atteint pas tout à fait le sixième posterieur de la tête. Funicule en massue très épaisse de 5 articles. Articles 2 à 6 très courts, plus du double plus larges que longs, plus larges que chez caca. Les 7 à io au moins un tiers plus larges que longs. Suture mésonotale obsolète. Pronotum aussi long que large (sans le col), déprimé, à profil presque rectiligne, obliquant légèrement jusqu'à la suture promésonotale, à partir de laquelle le profil du mesoépinotum, également rectiligne, s'incline au contraire légèrement vers l'arrière. Face déclive droite et bordée. Vue de dessus, la face basale de l'épinotum est bien plus étroite que le mésonotum, et n'est pas bordée. L'écaille est épaisse mais moins que chez cœeca, tronquée derrière, arrondie d'un côté à l'autre devant. Vue du haut, elle est le double plus large derrière que longue au milieu. Le sommet est arqué, un peu plus large que la base. Postpétiole moins haut que l'écaille, aussi large derrière que long, plus court que le segment suivant.

\section{Congo belge : Yambuya, 26-XI ( $\mathrm{D}^{\mathrm{r}}$ Bequaert, $\mathrm{n}^{\circ}$ 82).}




\section{5. - Sima Tesmannii Strtz.}

우 (non décrite). Long. : $5 \mathrm{~mm}$. Couleur, sculpture et pilosité de l'ø̧. Tête $^{\mathrm{I}} / 2$ fois plus longue que large, plus large et assez arrondie en arrière. Les yeux au tiers antérieur environ en ovale, courts, longs comme l'intervalle qui les sépare du bord antérieur. L'épistome a un lobe rectangulaire assez fort, dont le bord antérieur est faiblement trisinué. Le scape est beaucoup plus court que l'espace qui le sépare du bord postérieur de la tête. Funicule en massue comme chez l'ouvrière. Les mandibules sont fortement coudées à leur base, le reste du bord externe presque droit. Thorax aussi large que la tête. La face déclive de l'épinotum est perpendiculaire à la face basale, celle-ci aussi large que longue. Le petiole est aussi large derrière que long et légèrement plus large que l'épinotum. Le dessous porte une forte lame verticale arrondie aux angles comme chez $S$. Mayri For. Le postpétiole est en ovale transversal plus large que le pétiole et presque aussi large que le segment suivant. Fémurs très comprimés.

Très voisin de S. Mayri For. Cette dernière a l'épistome plus distinctement denté.

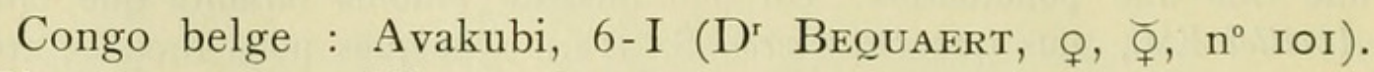
[Plante myrmécophile, $\mathrm{n}^{\circ}$ i 803.]

\section{6. - Sima encephala n. sp.}

․ Long. : $5.5 \mathrm{~mm}$. Rouge testace. Métanotum brun. Gastre un peu rembruni. Assez luisante. Tête mate, finement rugueuse en avant, plutôt réticulée dans le tiers postérieur. Reste du corps finement chagriné, le pronotum un peu plus nettement. Des poils jaunes assez courts sur les mandibules et le bord de l'épistome. Une pubescence clairsemée sur les appendices, la tête et le gastre. Tête rectangulaire, le double plus longue que large, les côtés parallèles, le bord postérieur droit, légèrement arrondi aux angles. Les yeux sont aussi longs que le tiers des côtés de la tête et sont placés vers le tiers postérieur. L'ocelle latéral est un peu plus rapproché de l'ocelle opposé que de l'œil. Les arêtes frontales atteignent la hauteur du bord antérieur des yeux. Elles sont subparallèles et très espacées. Les lobes frontaux plus rapprochés se touchent presque à leur base. Épistome convexe, non prolongé entre les lobes frontaux, formant devant un lobe à bord de six dents inégales, les externes plus fortes. Mandibules striées rugueuses coudées vers la base, le bord terminal aussi long que le bord interne à 5 dents. Le scape couché latéralement dépasse d'un quart de sa longueur les côtés de la tête. Articles 2-9 du funicule plus larges que longs, le ro aussi long que 
large, le i I aussi long que la somme des deux précédents. Tous s'épaississent de plus en plus. Thorax un peu plus large au milieu que la tête. Pronotum arrondi, sur le même plan que la face basale de l'épinotum au-dessus desquels le mésonotum et le scutellum forment une légère convexité. Les deux faces de l'épinotum subégales formant ensemble un angle très arrondi. Le pétiole vu de dessus parait fusiforme, plus du double plus long que large au milieu, bordé, les faces latérales planes, le dessus convexe, le pédicule assez court. Postpétiole en ovale transversal, aussi large que la longueur du pétiole, presque le double plus large que long; le dessous des deux articles inerme. Gastre plus large que le thorax, un peu plus large que le postpétiole. Le fémur postérieur étendu atteint le milieu des côtés du postpétiole.

\section{Sénégal : Saint-Louis (leg. Claveau).}

\section{7. - Melissotarsus major n. sp.}

ఫ઼. Long. : 2 6-3.3 mm. Tête et thorax rouge-brun. Appendices et abdomen jaune terne un peu brunâtre. Tête lisse, assez luisante, avec des points allongés espacés et des stries longitudinales vers le bord occipital, devant de la tête faiblement chagriné. Une vingtaine de fortes stries s'allongent sur le thorax. Abdomen lisse très finement réticulé, d'un aspect graisseux, plus luisant vers la base. De longs poils irréguliers, clairsemés, plus abondants sur le milieu de l'épistome, les mandibules, les scapes et les pattes. Pubescence rare et très courte.

Tête comme chez $M$. Beccarii Em., plus étroite devant. Yeux ovales de 30 facettes environ, plus longs que l'espace qui les sépare du bord antérieur de la tête. Mandibules à bord terminal irrégulier avec ou sans forte dent apicale. Le scape atteint l'œil. Le $2^{\mathrm{e}}$ article du funicule aussi long qu'épais à l'extrémité. Le $3^{\mathrm{e}}$ article quatre à cinq fois plus large que long. Les métatarses moyens sont un peu plus longs que larges (plus longs que chez Beccarii et moins que chez Emeryi), mais pas plus larges que les tibias. Pétiole un peu plus étroit que chez Beccarii. Postpétiole en ovale transversal deux fois plus large que long, bien plus volumineux que chez pilipes SANTs.

Diffère des autres espèces par sa grande taille.

Congo belge : Penghe, i3-II (Dr Bequaert, nº 125).

\section{8. - Crematogaster (Decacrema) solenopsoïdes EM. st.} flavida MAYR var. flaviscapis n. var.

ఫ̛. Long. : 2.8-3 mm. Jaune brunâtre clair, tête et abdomen plus foncés, 
appendices, y compris le scape, jaunes. Pilosite dressée rare et très fine; manque presque sur les appendices. Pubescence clairsemée et courte. Luisante, lisse avec de petits points piliferes très espacés. Quelques fines stries autour des fossettes antennaires, les côtés de l'épinotum et le fond du sillon mésonotal.

Tête à peine plus longue que large, le bord postérieur droit, au tiers moyen avec les angles très arrondis. Les yeux assez plats, grands comme le quart des côtés, en occupent le milieu. Le sillon frontal atteint le milieu de la tête. Aire frontale plus longue que large. Épistome peu convexe. Le scape atteint le quart postérieur de la tête. Articles 3 à 6 du funicule bien plus larges que longs. La bordure de la face déclive du mésonotum se prolonge sur sa face basale. Épines aussi longues que la moitié de l'intervalle de leur base. Pédicule à peine plus large que long, fortement denté en dessous.

\section{Congo portugais : Landana, I 5-VIII (Dr Bequaert, $\mathrm{n}^{\circ}$ I 4 ).}

\section{Var. gallarum SANTS.}

Cette variété fait passage au type de l'espèce, mais la pilosité dressée fait presque défaut sur ses appendices. Les yeux sont convexes et la carène médiane du mésonotum n'est pas très constante, toujours très faible, parfois très distincte

\section{9. - Crematogaster (Decacrema) solenopsoïdes Eм. st. costeboriensis $n$. st.}

б̆. Long. : $2 \mathrm{~mm}$. Brun foncé, gastre brun-noir, mandibules, antennes et tarses roux brunâtre. Devant des joues méso- et épipleuse faiblement strialé, le reste lisse. Pilosité clairsemée, assez abondante sur le thorax et surtout sur les appendices.

Tête un peu plus longue que large, convexe latéralement, les yeux plats. Le bord postérieur presque droit, les angles arrondis. Aire frontale et sillon frontal obsolètes. Épistome peu convexe, largement arrondi en arrière, le bord antérieur transversal, subtronqué et frangé. Le scape atteint le cinquième postérieur. Articles 3 à 6 du funicule plus larges que longs, mais moins larges que chez flavidula. Promésonotum convexe. Bords de la face déclive du mésonotum ne se prolongeant pas sur sa face basale. La face basale de l'épinotum bordée de côté, $2^{x} / 2$ fois aussi large que longue au milieu, elle passe à la face déclive par une convexité sagitale et une concavité transversale. Les épines sont un peu plus longues que le tiers de leur intervalle. Pédicule trapézoïdal; ses angles antérieurs et postérieurs subdentés; 
au-dessous une dent presque horizontale. Postpétiole un peu moins large que le devant du pétiole. Du reste comme solenopsoïdes Ем.

Côte d'Ivoire : région de San-Pedro (C. Phore).

Reçue du Muséum de Paris; je l'avais confondue avec le type.

IO. - Crematogaster (Sphaerocrema) rugosa ANDRÉ.

Congo belge : Avakubi, 3-I ( $\mathrm{D}^{\mathrm{r}}$ Bequaert, $\mathrm{n}^{\circ}$ io6).

Var. rugaticeps n. var.

Diffère du type par la sculpture de la tête plutôt grossièrement réticulée que striée en long avec le fond de la sculpture ponctué. Gastre et pédoncule (les deux nœuds) brun rougeâtre. La tête est plutôt plus longue que large et le scape la dépasse légèrement.

Cameroun : Molundu (leg. Reichensperger).

Var. nigriventris $\mathrm{n}$. var.

Diffère du type par l'abdomen entièrement noir. La tête et une partie du thorax sont au contraire d'un brun-noir ou rouge sombre. Les mandibules et les joues rouge foncé. La sculpture comme chez le type de l'espèce; la tête est aussi longue que large, plus longue que chez le type, les épines un peu plus longues, du reste semblable.

Côte d'Ivoire: Dimbroko (Le Moult).

II. - Crematogaster (Crematogaster) Meneliki FoR. st. proserpina SANTS. var. pluton n. var.

D'un brun-marron foncé; derrière de la tête, scapes, massue des antennes, les tibias et souvent l'extrémité du gastre plus obscur. Épines courtes comme chez proserpina, avec la même sculpture. Fait passage au type de l'espèce, dont il differe par ses épines courtes et divergentes, le devant du mésonotum porte une légère carène médiane. Le pétiole, en trapèze arrondi, est concave dessus.

Congo belge : Zambi, 2-VIII (Dr Beguaert, $n^{\circ}$ I). 
I2. - Calyptomyrmex (Dicroapsis) cryptocerus Ем. var. laevidens n. var.

б. Diffère du type par ses mandibules lisses et luisantes (striées chez le type) avec des points espacés. Pour le reste, comme la description du type.

Congo belge : Yambuya, 26-XI (Dr Bequaert, n 83).

I3. - Tetramorium coloreum MAYR var. postpetiolata n. st.

ф. Un peu plus petit que le type, les arêtes frontales un peu moins espacées. Le postpétiole lisse et bien plus large que long (strié et aussi long que large chez le type); il est aussi plus clair, aussi clair que le gastre. La face postéro-supérieure du pétiole est, au contraire, plus rugueuse. Thorax plus rougeâtre, du reste comme le type.

Congo belge : Penghe, 25-I (Dr Bequaert, nº i 33 ).

\section{I4. - Xiphomyrmex Muralti For.}

Congo belge: Penghe, 27-I ( $D^{r}$ Bequaert, no i i 6$)$.

Xiphomyrmex Muralti For. var. trilineata n. var. $(=\mathbf{X}$. Mu= ralti SAnts., Boll. del Lab. di Zool. gen. e Agrar. della R. Scuola sup. d'Agric. Portici, vol. VIII, p. 368, 1914.

Cette variété diffère du type, qui n'a qu'une seule ride médiane, par la présence de deux autres rides placées chacune entre la médiane et l'arête du scrobe, un peu plus près du milieu. Elles sont aussi longues et parallèles à la médiane, c'est-à-dire qu'elles s'étendent du bord antérieur de l'épistome au bord occipital. La tête est un peu plus large et il y a quelques traces de rides sur le devant du pronotum; pour le reste, comme le type.

Gold Coast : Aburey (Silvestri).

\section{I5. - Strumigenys (Cephaloxis) glanduscula n. var.}

ఫ. Long. : 2-2.2 mm. Jaune roussâtre, mandibules plus roussâtres, à bord denté brunâtre. Tête et thorax finement réticulés, ponctués et mats. Occiput 
et épaules plus luisants. Face antérieure du pétiole plus légèrement réticulé. Reste du pédoncule, gastre et mandibules lisses et luisants. Base du gastre non striée. Pilosité courte, courbée, non clavée. Pilosité longue, faiblement clavée sur le gastre (plus faiblement que chez Escherichi For., avec la même disposition.

Tête un peu plus courte et plus échancrée derrière que chez Simoni Em., les côtés un peu plus convexes. Aire frontale allongée, imprimée et luisante. Épistome relativement plus grand que chez Escherichi, les mandibules plus courtes que chez cette dernière, plutôt comme chez Simoni Em., de même que les antennes Quand le scape est récliné, le bout du funicule atteint le milieu des mandibules. Le promésonotum forme une convexité allongée comme chez Escherichi FoR. La déclivité formée par le mésonotum, est très oblique et presque rectiligne sur le profil. La face basale de l'épinotum se relève brusquement derrière le sillon métanotal, pour former un plateau presque carré à côtés mousses. Les dents ou épines relativement petites et la face déclive est bordée par une bandelette membraneuse très étroite.

Pédicule du pétiole presque plus long que le nœud, celui-ci aussi large que long, arrondi au sommet. Une bandelette au-dessus de l'article dont la largeur ne dépasse pas la moitié de l'épaisseur du pédicule. Postpétiole aussi long que le nœud précédent, un quart à un tiers plus large que long (bien plus étroit que chez Escherichi). Bordé derrière et dessous d'une bandelette membraneuse aussi étroite que celle de l'article précédent.

Intermédiaire entre Simoni Em. et Lujae For. Mais le premier est plus petit et a le pédicule sculpté, de même chez Lujae, auquel manquent les bandelettes.

Congo belge : Yambuya, 25-II (Dr Bequaert, $n^{\circ} 79$ ).

\section{I6. - Technomyrmex hypoclinoïdes n. sp.}

ఫ. Long. : 2.5-2.6 mm. D'un jaune brunâtre terne, appendices plus clairs, la moitié distale du funicule rembruni. Très finement chagriné-réticulé. La tête et l'épinotum plus densément que le reste du thorax et celui-ci que le gastre. La tête et l'épinotum presque mats, le reste du thorax et surtout le gastre plus luisants avec un éclat graisseux. Une paire de longs poils sur l'épistome, le pronotum, l'épinotum et le gastre. Quelques poils moyens vers la bouche et l'abdomen. Pubescence rare, plus apparente sur le gastre.

Tête rectangulaire, un quart plus longue que large, un peu rétrécie dans le quart antérieur, les côtés un peu convexes, le bord postérieur transversal avec les angles brièvement arrondis. Les yeux ovales, peu convexes, aussi grands que le quart des côtés de la tête, en occupent à peu près le milieu. 
Sillon frontal nul. Aire frontale grande, faiblement limitee. Épistome médiocrement convexe, largement arrondi entre les courtes arêtes frontales, sans carène, le bord antérieur transversal profondément échancré au milieu, avec l'insertion d'un long poil de chaque côté de cette échancrure. Mandibules lisses, à bord externe très convexe, le bord terminal assez long de 6 à 7 dents subégales. Le scape atteint le bord postérieur de la tête. Articles 2 à 9 du funicule environ aussi épais ou un peu plus épais que longs. Le pronotum convexe, un peu plus large que long, a un gros point pilifère de chaque côté de son milieu. Le mésonotum continue sur le profil la courbe du pronotum, mais elle est moins convexe; il en est séparé par une suture distincte que renforce une légère crête transversale formee par le bord antérieur. Métanotum environ $2 \frac{1}{2}$ fois aussi large que long, les stigmates font légèrement saillie sur ses bords. Face basale de l'épinotum plus de quatre fois plus longue que la face déclive dont elle est séparée par une forte arête transversale, rectangulaire, un tiers plus longue que large, brusquement rétrécie au dernier tiers qui sont marqués, ainsi que les angles postérieurs, par de petits tubercules pilifères. La face déclive est fortement concave de haut en bas, surplombée par la crête de délimitation et bien plus large en haut qu'en bas. Le pétiole a une écaille inclinée, mais faisant saillie de toute son épaisseur au-dessus de son pédicule antérieur et dont le sommet est acuminé. Gastre allongé ne couvrant pas l'écaille.

Congo belge : Avakubi, 6-I ( $\mathrm{D}^{\mathrm{r}}$ Bequaert, $\mathrm{n}^{\circ}$ io3). [Plante myrmécophile, $\mathrm{n}^{\circ}$ I 796.]

Espèce très aberrante; son épinotum la fait ressembler à une Hypoclinea.

\section{7. - Plectroctena subterranea ARN. st. gabonensis n. st.}

б. . Long. : $9.5 \mathrm{~mm}$. Noire brunâtre; pattes et extrémité de l'abdomen plus rougeâtre; funicule noirâtre. Luisante, lisse avec une ponctuation plus fine que chez $P$. minor EM., et les côtés du thorax striolés comme chez cette espèce. La tête est relativement plus courte que chez minor. Les yeux plus petits mais plus grands que chez subterranea ARN. (d'environ 50 facettes). La distance qui les sépare du bord antérieur de la tête est moindre que leur longueur. Il s'en faut du double de son épaisseur que le scape atteigne le bord postérieur de la tête. Suture méso-épinotale indiquée par une légère impression transversale. Nœud du pédicule plus long que 
large. Le devant du postpétiole est bordé par un léger bourrelet présentant une impression postérieure. Le reste comme chez subterranea ARN. C'est une forme intermédiaire.

Gabon (F. Faure), igi4. Un exemplaire, au Muséum de Paris, provient également du Gabon.

Dans mon précédent travail publié dans cette Revue, volume VI, il s'est glissé quelques erreurs d'impression qu'il importe de relever ici :

Pages 232-233. Les variétés lugubris et marginiventris doivent se rapporter, comme variété, à Dorylus (Rhogmus) fuscipennis Eм., comme cela est indiqué dans le tableau de la page précédente, et non à fimbriatus.

Page 233, lige 6, au lieu de : forculatus $\mathrm{n}$. sp., lire : furculatus $\mathrm{n} . \mathrm{sp}$.

Page 239, ligne 21, au lieu de : $M$. radamoides, lire : st. radamoide.

Page $244,2^{\mathrm{e}}$ ligne du bas, au lieu de : Long. : 2.3-2.4 mm., lire : $23^{-24} \mathrm{~mm}$. 


\section{$2 \mathrm{BHL}$ Biodiversity Heritage Library}

1919. "Nouvelles fourmis du Congo belge du Musee du Congo belge, a Tervueren." Revue zoologique africaine 7, 79-91. https://doi.org/10.5962/bhl.part.15111.

View This Item Online: https://www.biodiversitylibrary.org/item/42145

DOI: https://doi.org/10.5962/bhl.part.15111

Permalink: https://www.biodiversitylibrary.org/partpdf/15111

\section{Holding Institution}

American Museum of Natural History Library

\section{Sponsored by}

Biodiversity Heritage Library

\section{Copyright \& Reuse}

Copyright Status: NOT_IN_COPYRIGHT

This document was created from content at the Biodiversity Heritage Library, the world's largest open access digital library for biodiversity literature and archives. Visit BHL at https://www.biodiversitylibrary.org. 